\title{
Language evolution research in the year 2020 A survey of new directions
}

\author{
Jonas Nölle \\ Centre for Language Evolution, University of Edinburgh, Edingburgh, U K \\ j.nolle@sms.ed.ac.uk \\ Stefan Hartmann \\ University of Düsseldorf, Düsseldorf, Germany \\ hartmast@hhu.de
}

Peeter Tinits

University of Tartu, Tartu, Estonia

peeter.tinits@ut.ee

\begin{abstract}
This introductory paper reviews recent advances in language evolution research and summarizes the contributions of the special issue "New Directions in Language Evolution Research" in the broader context of these developments. Specifically, we discuss the increasing role of multimodality and iconicity, the more integrative view of language dynamics that has arguably broadened the scope of language evolution research, and recent methodological innovations that allow for a more fine-grained study of e.g. typological distributions or behavioral patterns that can give clues to some of the key questions discussed in the field.
\end{abstract}

\section{Keywords}

language evolution - methodological advances - interdisciplinarity

\section{Introduction}

Research on language evolution is undoubtedly among the fastest-growing topics in linguistics. This is not a coincidence: While scholars have always been 
interested in the origin and evolution of language, it is only now that many questions can be addressed empirically. Researchers can now draw on a wealth of data and a multitude of methodological approaches that have been developed in the different disciplines that try to find answers to what has been called "the hardest problem in science" (Christiansen \& Kirby, 2003). Importantly, any theory of how language may have emerged requires a solid understanding of how language and other communication systems work. As such, the questions in language evolution research are not just manifold but also interface with key open questions in historical and theoretical linguistics: Where does human language come from and what makes it unique compared to animal communication systems? How do cognition, communication and transmission shape grammar? Which factors can explain linguistic diversity? How and why do languages change? To what extent is the structure of language(s) shaped by language-internal and external environmental factors?

The aim of this introductory article is to give a brief overview of current new directions in language evolution research that are taken to address these questions. As Dediu \& de Boer (2016) have noted, the field of language evolution research is currently coming of age, and has developed a rich toolkit of methods both for comparative research, which investigates the commonalities and differences between human language and animal communication systems, and for studying the cumulative cultural evolution of sign systems in experimental settings, including both computational and behavioral approaches (see, e.g., Tallerman \& Gibson, 2012; Fitch, 2017). In addition, large-scale typological studies have gained importance in recent research on language evolution (e.g., Evans, 2013; Blasi et al., 2016; Majid et al., 2018; Jackson et al., 2019). Here, we survey important recent developments in the field, and discuss how the papers collected in this special issue of Language Dynamics and Change can be seen as representative of these new directions. Note that the special issue is split into two volumes, which is why some of the papers summarized here will appear in the next issue of LDC.

Quo vadis language evolution research? An overview of new directions

The view that there has been a "gap" in language evolution research between the infamous ban of discussions relating to the emergence of language by the Societé de Linguistique de Paris in 1861 and an alleged resurgence of the field in 199os has long been identified as a myth (see, e.g., Fitch, 2010: 16). However, it is true that the field has seen a surge since the late zoth century, and it has 
evolved considerably over the past two decades. In this section, we briefly outline a number of major trends that are also taken up in the contributions to the special issue: the increasing importance of multimodality and iconicity in accounts of the evolution of language; taking into consideration contextual and environmental factors more thoroughly; and the growth of interdisciplinary approaches in both theory and method.

Regarding the relationship between theory and method, the question of causality has been discussed intensively. Various interacting factors have led to a shift in perspective here: First, the notorious problem of spurious correlations has been pointed out in various papers (e.g., S. Roberts \& Winters, 2012, 2013). Second, the "replication crisis" (e.g. Open Science Collaboration, 2015) has affected virtually all domains of empirically-based scientific inquiry, including linguistics, and third, language evolution research often relies on very indirect evidence. This makes it attractive not only for theoretical "just-so stories" but also for both far-reaching and far-fetched empirical hypotheses, which increases the problem of spurious correlations and makes the sanity checks that have been called for in the wake of the replication crisis all the more necessary.

The key question, then, becomes: Which hypothesized causal relationships are robust and what kind of evidence can support those causal links? Many of the variables discussed in language evolution research are causally linked and interact with each other in different ways. Therefore, in order to avoid the problem of spurious correlations, a new approach, which S. Roberts (2018a) terms the 'maximum robustness approach', is gaining momentum. Instead of focusing on simple relationships, e.g., environmental variable $\mathrm{X}$ correlating with linguistic trait $Y$, the idea is to systematically construct more complex and coherent causal graphs (Pearl, 2009) that are based on all the available evidence as well as hypothesized links between multiple interrelated variables. CHIELD, a novel database for hypotheses in language evolution, allows for exploring such graphs and identifying complementary or conflicting relationships as well as empirical gaps that lack evaluation, which can help design empirical research addressing those issues and uncovering actual causal mechanisms (S. Roberts, 2018a, S. Roberts et al., 2020).

\subsection{Multimodality and iconicity}

The modality where language originated has been hotly debated: According to the "gesture-first" view, "language evolved initially from manual gestures with vocal elements gradually added" (Corballis, 2012: 383; see also, e.g., Corballis, 2003, Tomasello, 2008, Arbib, 2012, among others; see McNeill, 2012, for an overview and criticism). The "speech-first" view defended by e.g. Dunbar (1996) 
and MacNeilage (2008), by contrast, argues that language must always have existed in the vocal-auditory modality given its present-day dominance (see, e.g., Zlatev et al., 2017:453). These different theories, which go back even further than the works cited here (see, e.g., Fitch, 2010 for a historical overview), are now increasingly replaced by more nuanced and fine-grained views that argue for a complex interplay between auditory and visual channels in the early multimodal beginnings (e.g. Kendon, 2017; Perlman, 2017). For example, regarding the visual-spatial modality, the idea of "pantomime-first" has been put forward as "a theoretical proposal that is arguably distinct from both gesture-first and multimodal-first” (Zlatev et al., 2017: 473; also see Żywiczyński, Wacewicz \& Sibierska, 2018).

In line with the strongly empirical outlook of modern language evolution research, a number of studies have set out to test the predictions of those different theories empirically (e.g., Zlatev et al., 2017; Macuch Silva et al., 2020). Across modalities, the key role of iconicity has received increased attention (e.g., Perniss, Thompson \& Vigliocco, 2010; Perniss \& Vigliocco, 2014; Dingemanse et al., 2015; Pleyer et al., 2017) with the similarities and differences between modalities in the affordances for iconicity being highlighted (Perlman et al., 2018) and methodological innovations developed to assist systematic study (Motamedi et al., 2019a).

Importantly, these theories go in tandem with a more general development in the language sciences noted by e.g. Wacewicz \& Żywiczyński (2017), namely that face-to-face interaction represents "the core ecological niche for language" (Torreira, Bögels \& Levinson, 2015: 1). Language is therefore more and more regarded as a multimodal phenomenon. In particular, the role of gesture has received increasing interest (see, e.g., Müller et al. [eds.], 2013). The affordances of the gestural modality for grounding communication systems have been investigated quite extensively. On the one hand, experimental studies have shown how participants develop new gestural communication systems from scratch (Galantucci, Garrod, \& G. Roberts, 2012; Motamedi et al., 2019b). On the other hand, the emergence of new sign languages such as Nicaraguan Sign Language (NSL) and Al-Sayyid Bedouin Sign Language has supplemented theories of language emergence with natural data.

Carla Hudson Kam and Oksana Tkachman add to this line of research by investigating the role of interpretability. In particular, they focus on the use of spatial modulations to encode concrete spatial relations on the one hand and more abstract grammatical relations on the other. Starting from the finding that the way entities are placed in signed space seems to facilitate the way grammatical relations, but not actual spatial ones, are understood in NSL, they conduct a series of experiments with hearing non-signers—adults and children — testing 
the hypothesis that the locative use of space is less directly interpretable as it requires more interpersonal coordination and thus takes more time to conventionalize. In a first experiment, participants were presented with a short narration mentioning two characters, with a pronoun referring back to one of them. Depending on the condition, the narrator used no gesture, the same gesture for the character and the pronoun referring to this character, or different gestures for the character and the pronoun. Hudson Kam and Tkachman's results show that both children and adults interpret pronouns differently based on co-speech gestures. In a second experiment, participants also watched short narratives involving two characters, with the narrator producing co-referential localizing gestures indicating the spatial location of the two characters (left vs. right). In a picture selection task, participants were then asked to indicate which picture best described the story they had heard. They were shown two versions of the same scenario that differed in the relative spatial location of the characters. While the adults showed some limited consistency in their interpretation, the children did not. They conclude that adults and older children, even if not familiar with a signed language, take in spatial information conveyed via co-speech gesture while younger children do not, possibly because the interpretation of spatial information is quite demanding in that it requires inferences about spatial configurations as well as about perspectivation.

Whereas Hudson Kam \& Tkachman are concerned with integral features of sign languages that do not arise in spoken language, the study by Katie Mudd, Connie de Vos and Bart de Boer uses data from an emerging sign language for modelling language persistence. Building on a mathematical model of sign language persistence (Aoki \& Feldman, 1991), they inform their agent-based model with real-world data. In particular, they investigate the impact of extralinguistic factors such as the number of deaf individuals in the population, the number of hearing carriers of the deaf allele, population size, and mating patterns. In their model, the sign language does not persist in a small population, while the proportion of deafness in the population only leads to non-persistence if it reaches an extremely low threshold. However, they show that for both results, there are counterexamples in existing emerging sign languages, and they discuss additional factors that may be necessary to explain the persistence of sign languages.

\subsection{An integrative approach to language dynamics}

Another important trend in language evolution research is that its scope has extended quite significantly. This can partly be seen as a by-product of a different but related development, namely the increasingly widespread view of language as a complex adaptive system, i.e. a system whose global properties 
arise from multiple independent interactions on the local level (e.g., Steels, 200o; Beckner et al., 2009; Kirby, 2012), which entails a more unified approach to the ontogentic, phylogenetic, and "glossogenetic" (Hurford, 1990) dimensions of language dynamics. This is why the term "language evolution" is sometimes used without terminological consistency, as Haspelmath (2020) notes: On the one hand, it can refer to the biological evolution of the biological capacity for language (called "linguisticality" by Haspelmath), on the other hand, it can refer to the cultural evolution or diachronic change of particular language systems (also see Mendívil-Giró 2019, Hartmann in press). While Haspelmath (2020) argues that "[t]he comparison of languages does not lead to immediate insights about human linguisticality", various scholars have defended the view that investigating present-day distributional patterns can yield valuable clues to the emergence of language. For instance, Heine \& Kuteva (2007, 2012) argue that "typological generalizations on language structures" (Heine \& Kuteva, 2007: 55) can be used to reconstruct the "genesis of grammar" with the help of grammaticalization theory. In a similar vein, Bybee (2010: 202) emphasizes the relevance of investigating historical language change for understanding language evolution: "there is every reason to suppose that the very first grammatical constructions emerged in the same way as those observed in more recent history". However, note that both Bybee and Heine \& Kuteva hedge their hypotheses carefully, emphasizing that their accounts can only give clues to the origins of grammatical units, not language per se. However, as e.g. Antonio Benítez-Burraco points out in his paper, investigating both typological and grammaticalization patterns can point to environmental and social driving forces in cultural evolution, which in turn can indeed be relevant for understanding the origins of the human capacity for language.

While Haspelmath's (2020) distinction between "evolution of linguisticality" and "evolution of languages" reminds us that both biological and cultural factors are at play in language development, it is also important to keep in mind that we cannot clearly tease apart the emergence of the language capacity from the cultural evolution of language(s). This point is made in a particularly forceful way by coevolutionary models, which assume that the evolution of language, culture, and biological features interact (see, e.g., Evans, 2013; Kirby, 2017). In the present special issue, Benítez-Burraco makes a strong case for this hypothesis. Arguing against the view that "the evolution of language" on the one hand and "the evolution of languages" on the other can and should be teased apart, he points to recent evidence according to which (i) human cognition might not be as uniform as is usually assumed and might have changed over our recent history, and (ii) the structure of languages is partly shaped by social, cultural, and environmental factors. He therefore argues for an eco- 
evo-devo account of language evolution, emphasizing the role of human selfdomestication, i.e. the emergence of traits typically associated with domestication in biological evolution, but without external influence.

Studies investigating historical language change have shown that methods from evolutionary biology can be fruitfully applied to the reconstruction of language history (see, e.g., Gray, Drummond \& Greenhill, 20o9, Syrjänen et al., 2016, Bouckaert, Bowern \& Atkinson, 2018). Additionally, theoretical approaches like Croft's (2000) evolutionary approach to language change or Blevins' (2004) and Ritt's (2004) evolutionary approaches to phonology have shown the potential of mutual cross-fertilization between linguistics and evolutionary theory. Language itself has come to be viewed more holistically. On the one hand, as pointed out in Section 2.1, language is increasingly seen as a fundamentally multimodal phenomenon. On the other hand, despite its undisputed special status, language is no longer seen as the "odd one out" among our cognitive and cultural traits. Instead, an integrative view sees language as bearing important commonalities with other phenomena, which is why phenomena as different as the construction of paper airplanes (Caldwell \& Millen, 2008), the evolution of a collaborative art project (Müller \& Winters, 2018), diachronic changes in ancient coin designs (Pavlek, Winters \& Morin, 2019), and the evolution of language(s) can be investigated in a shared theoretical framework. The 2016 founding of the Cultural Evolution Society bears witness to the appeal of such an integrative approach to language, cognition, and culture.

These ideas are of course not entirely new. Among others, Humboldt (1836) has already argued for a close connection between language, cognition, and culture, even though many of his ideas are by now outdated, to say the least (e.g., the assumption that the complexity of a language reflects a nation's degree of civilization). What is (relatively) new, however, are many of the methods that can now be used to study these relationships in more detail. This arguably also entails a more thoroughly interdisciplinary outlook: While language evolution research has been a highly multi-disciplinary field from the outset, it could be argued that this interdisciplinarity has often consisted in combining insights from studies that themselves largely remained within disciplinary boundaries. Many more recent studies, however, cross these boundaries by taking a strongly interdisciplinary approach already in the design of their studies. For example, Dediu \& Levinson $(2013,2018)$ argue that Neanderthals had vocal language on the basis of archaeological as well as genetic evidence, and Tylén et al. (2020) combine the analysis of archaeological findings with behavioral experiments to investigate the evolution of symbolic communication. 
The interpretation of archaeological findings for studying language evolution entails some obvious challenges: For instance, it is a matter of debate what kinds of archaeological records constitute evidence for symbolic or even linguistic behavior (see, e.g., d'Errico \& Vanhaeren, 2009; Botha, 2012). This is why Cory M. Stade proposes to use Theory of Mind (ToM) as a proxy for language ability, rather than relying on symbolism. She argues that ToM might be better identifiable in the archaeological record as certain social behaviors have been shown experimentally to require theory of mind. She mentions a number of advantages, e.g. the strong correlation that has been found between ToM and language, which has led to the widely-shared assumption that both have co-evolved (see Woensdregt, Cummins \& Smith, forthc. for a computational model). Stade's study is particularly relevant in light of recent discussions about ToM in general (see e.g. François \& Rossetti, 2020; Sulik \& Lupyan, 2020) and may give rise to methodological discussions about how ToM can be defined and operationalized more thoroughly.

Representing the explorations of interdisciplinary connections, Gareth Roberts and Betsy Sneller make a case for increased collaboration between sociolinguists and language evolution researchers by comparing the five foundational problems in language change (Weinreich, Labov \& Herzog, 1968) and the four foundational questions in ethology (Tinbergen, 1963). The paper discusses resemblances between sociolinguistic and cultural evolutionary approaches that both deal with population-level problems like the spread and propagation of features as well as opportunities for integrating them in a mutually informative way. Roberts and Sneller further show how experimental paradigms that are usually used to study language evolution can be used to test sociolinguistic theories of language change, and how the study of natural language change can in turn inform experiments in cultural language evolution. An interesting point that they note is that the questions driving language evolution research today were already embraced by Weinreich, Labov \& Herzog in their formulation of a general science of language change.

\subsection{Methodological innovations}

In his recent detailed review, Fitch (2017) already lays out the wide varieties of methods and interdisciplinary approaches that are now available to researchers from various fields to study the components of the human language faculty, drawing on comparative biology, neuroscience, paleontology and genetics. Similarly, there has been an explosion in experimental studies addressing the cultural evolution of linguistic structure in the lab, ever since pioneering studies showed that both the grounding of communication systems from scratch in horizontal interaction (Galantucci, 2005), and the verti- 
cal cumulative cultural evolution of language over generations (Kirby, Cornish \& Smith, 2008) can be simulated with human participants in controlled settings. These approaches are now referred to as 'experimental semiotics' (Galantucci, Garrod \& G. Roberts, 2012) and 'iterated learning' (Kirby, Griffiths \& Smith, 2014). They initially served as empirical validations of early computational models that suggested linguistic structures could emerge through interaction and transmission (e.g., Steels, 1995; Kirby \& Hurford, 2002). However, since then, studies using artificial communication systems have matured into a range of paradigms that have been used to study the emergence and evolution of linguistic structure and the pressures shaping it (Tamariz, 2017). General observations include different pressures for simplicity/learnability and expressivity/informativeness that can result from horizontal interaction or vertical transmission (Kirby et al., 2015; Motamedi et al., 2019b; Carr et al., forthc.). Other recent studies have scrutinized in what way communication systems are sensitive to properties of the signaling space (Little, Eryilmaz \& de Boer, 2017; G. Roberts, Lewandowski \& Galantucci, 2015), properties of the meaning space (Carr et al., 2017), the referential context (Winters, Kirby \& Smith, 2015, 2018; Tinits et al., 2017), factors related to the mode of communication (Nölle et al., 2018), or information-theoretic principles (Kanwal et al., 2017, Stevens \& Roberts, 2019).

This includes, for instance, how languages order semantic constituents (e.g., Goldin-Meadow et al., 2008; Schouwstra \& de Swart, 2014; Özçalışkan, Lucero \& Goldin-Meadow, 2016; Meir et al., 2017) or modifiers within the noun phrase (Culbertson \& Adger, 2014; Martin et al., 2019) as well as how the social environment, e.g., group size (Raviv, Meyer, Lev-Ari, 2019) or social biases (Sneller \& Roberts, 2018; G. Roberts \& Fedzechkina, 2018), affect the evolution of linguistic systems. In this way, artificial language learning, communication and transmission studies have become powerful tools to study the mechanisms underlying linguistic universals and diversity more generally. This point is echoed by G. Roberts and Sneller, who argue that artificial language experiments could have a significant impact on sociolinguistics and studies of language change.

Besides cross-fertilization with other linguistic subfields, multidisciplinary approaches to language evolution are now commonplace. This is in part due to the realization that language does not evolve in a void but is rather "the product of a multiscale communicative niche construction process at the intersection of biology, environment, and culture" as Coupé et al. (2019:1) formulate it. There is now increasing evidence that a large number of features that vary widely across languages, such as specific speech sounds (e.g., Everett, Blasi \& Roberts, 2015; Moisik \& Dediu, 2017) or lexical or semantic categories (e.g., 
Brown \& Lindsey 2004; Regier, Carstensen \& Kemp, 2016) might not just reflect arbitrary cultural 'choices', but are rather motivated by and sensitive to the specific local physical, sociocultural or technological environment (see Lupyan \& Dale, 2016 for an overview). While this kind of 'linguistic adaptation' was initially suggested due to observed correlations, such as the inverse relationship between morphological complexity and population size (Lupyan \& Dale, 2010), such findings are now corroborated by phylogenetic evidence suggesting that the historical diversification of language families is better explained by environmental factors such as a climate rather than neutral drift (Bentz et al., 2018; Hua et al., 2019). An ambitious paper by Blasi et al. (2019) illustrates just how rigorous and transdisciplinary more recent approaches to linguistic adaptation have become: Combined results from biomechanical modeling of bite configurations with phylogenetic analyses of language change and ethnographic data suggests that changes in diet during the neolithic revolution have affected the distribution of labiodental sounds such as [f] and [v]. These phonemes are significantly easier to produce with an overbite that resulted from the transition into agriculture leading to a softer diet.

Such endeavors are to some extent enabled by a recent surge in large-scale databases enabling what Bickel (2015) calls 'distributional typology', a datadriven comparative approach that addresses the what, where and why questions regarding language structure with statistical techniques. With Glottolog (Hammarström, Forkel \& Haspelmath, 2019), linguists have access to an accurate classification for approximately $6500-8000$ linguistic varieties including information on (sub-)families and dialects (see Hammarström, 2016, for an introduction and overview) and there are now many specialized databases for linguistic features (e.g., waLs, Dryer \& Haspelmath, 2013), phoneme inventories (PHOIBLE, Moran \& McCloy, 2019), basic vocabulary word lists (ASJP, Wichmann, Holman \& Brown, 2018) as well as databases that allow linking linguistic and cultural or environmental data (D-PLACE, Kirby et al., 2016). These and other data sources (see http://languagegoldmine.com/ for a comprehensive list) are now increasingly used to investigate general patterns found across languages, such as systematic sound-symbolic meaning associations in the lexicon (Blasi et al., 2016) or universals and variation in colexification of emotion words across thousands of languages (Jackson et al., 2019). Similarly, an evergrowing number of corpora enables detailed synchronic and diachronic analyses of single languages and allows characterizing individual- and populationlevel dynamics that are involved in historical language change (e.g. Petré \& Van de Velde. 2018). Recent work using English corpora has, for instance, addressed to what extent sensory vocabulary reflects communicative efficiency across semantic domains (Winter, Perlman \& Majid, 2018). 
The paper by Andres Karjus, Richard A. Blythe, Simon Kirby, and Kenny Smith represents another example of this line of research. They discuss how large-scale corpora can help understand language dynamics and change. Previous work in the field of "culturomics" (e.g., Michel et al. 2011) has been heavily criticized because "these approaches often present sweeping accounts of the historical development of words and concepts with little or no attention paid to important linguistic clues and principles of good science." (Brezina, 2018: 224) Unlike those previous works, the "topical-cultural advection model" that Karjus et al. propose aims at teasing apart different explanatory factors in order to separate random from systematic effects. Two case studies show that the model is able to capture topical effects in frequency changes of linguistic elements over time.

With the availability of rich data sources, it is tempting to simply search for cross-sectional patterns. For instance, economists have recently begun linking linguistic features such as future-tense marking or pronoun-drop to economic behavior (e.g., Chen, 2013; Kim, Kim \& Zhou, 2017; Feldmann, 2019). However, one argument for the 'maximum-robustness' approach discussed in section 2 above is that such large-scale correlational studies are not well-suited to make causal claims, since relationships between linguistic and languageexternal variables are often complex and confounded (e.g., due to historical or geographic relatedness of cultural traits). It has therefore been argued that experiments or case studies are a better way for verifying such relationships (S. Roberts, Winters \& Chen, 2015; S. Roberts, 2018b).

This is exemplified by the relationship between spatial language and the environment: For a long time, field linguists have speculated that the way in which speech-communities reason and talk about spatial relations could be dependent on the local topographic environment (e.g., Wassmann \& Dasen, 1998). However, large-scale surveys did not find simple relationships between spatial linguistic strategies and environmental factors such as climate or subsistence style (Majid et al., 2004). Only more recently, detailed individual case studies and comparisons between environments and speech-communities have found that social and environmental variables such as second language, subsistence style or local topography do indeed explain some of the variance in spatial linguistic strategies (e.g., Bohnemeyer et al., 2015; Palmer et al., 2017; Shapero, 2017). However, it is hard to disentangle the exact causal relationships between such variables. Magnus Pharao Hansen and Carolyn O'Meara therefore argue for an integrated approach to the adaptation of spatial language, through a combination of ethnolinguistic fieldwork and historical studies of language change. They provide a detailed framework for selecting linguistic varieties with regard to their grammatical differences, ambient climate and 
landscape features, and available historical data that can be used to reconstruct whether an adaptation to environmental factors has taken place in the development of these varieties. Observations from real linguistic data could then be tested with computational models (see, e.g., Spranger, 2016) or human subject experiments testing the impact of the environment on linguistic conventions (e.g., Nölle et al., 2020a).

Russell Richie, Matthew L. Hall, Pyeong Whan Cho, and Marie Coppola provide another example that shows why it is important to address hypothesized causal relations with a mixed-methods approach. Building on previous work from experimental semiotics as well as natural observation and computational modeling, they investigate the role of social network dynamics in language emergence. Using a dyadic gestural communication task, they investigate whether conventionalization differs between two conditions, one with a richly-connected and one with a sparsely-connected communicative network. In the richly-connected communicative network, each member of a group of four participants gets to interact with every other member, while in the sparse condition, one member is involved in all dyads, thus serving as a "hub". They show that conventionalization is greater and grows more quickly in the richly-connected network when measured per unit time, i.e. per round in the experiment; however, the effect disappears when measured per interaction (the richly-connected network condition contains more interactions than the sparsely-connected network condition). A possible explanation is that there are different mechanisms of conventionalization at work: In the sparse network, the person that serves as a "hub" can set a standard that other participants tend to converge on; while in the rich network, participants have to negotiate whose utterances they will converge on, which is why participants in richlyconnected networks do not conventionalize referring expressions faster, per interaction, than those in sparsely-connected networks. In contrast, in a rich network with more interactions, conventionalization happens faster than in the sparse network.

Another promising avenue is the use of novel technology. For a long time, experimenters had to navigate a trade-off between experimental control and ecological validity. However, with the availability of new methodologies like immersive virtual reality (VR), it is now becoming clear that it is possible to design tasks that are both tightly controlled and naturalistic (Peeters, 2019). VR holds a lot of potential for investigating cases like spatial language, e.g., by comparing spatial linguistic strategies across simulated large-scale environments that resemble those that have been speculated to affect natural languages (see Nölle et al., 2020b). As discussed in section 2, it is this interdisciplinary approach that can help address causality and model the actual 
mechanism underlying cross-linguistic variation more rigorously and incrementally. Lastly, artificial intelligence researchers have recently begun modeling the emergence of compositional structure using deep reinforcement learning algorithms with a similar approach to early evolutionary linguistic models (Kirby \& Hurford, 2002). In such models, compositionality emerges in multi-agent learning environments with different signal and meaning spaces to enable coordination (Lazaridou, Peysakhovich \& Baroni, 2017; Mordatch \& Abbeel, 2018; Choi, Lazaridou \& de Freitas 2018). While this trend in AI has begun independently, recent work is beginning to converge with findings in language evolution research, for instance by introducing generational transmission as a cultural evolutionary mechanism (e.g., Cogswell et al., 2019; Ren et al., 2019).

\section{$3 \quad$ Conclusion}

The papers in this special issue show that the field of language evolution research has evolved substantially in recent years. Various new approaches, both theoretical and methodological, have been developed that shed new light on the key questions of language evolution research. But the new directions we have sketched here have not only influenced the way we address these questions. Instead, we have also seen a shift in focus regarding the research questions that are asked: Traditionally, the question of when, how, and why the capacity for linguistic communication evolved has been at the center of language evolution research. As we have seen, these questions are still important: For instance, archaeological and genetic evidence keeps providing new insights on the antiquity of language. More and more, however, the focus has shifted to uncovering the co-evolutionary dynamics of language, cognition, and culture. This has also entailed a reconceptualization of the scope of language evolution research, which now involves the study of language dynamics and change more generally from a cultural evolutionary perspective. The contributions to this special issue reflect this tendency. They also show the strongly interdisciplinary outlook of modern language evolution research in discussing how insights from previously neglected fields can contribute to our understanding of how language evolved and continues to evolve. 


\section{Acknowledgments}

The papers collected in this special issue partly go back to presentations at the workshop "New Directions in Language Evolution Research" at the 51st Annual Meeting of the Societas Linguistica Europaea in Tallinn. We are grateful to all participants for stimulating presentations and discussions. Also, we would like to thank the LDC editors Simon Greenhill and Jeff Good for their guidance throughout the editorial process as well as the anonymous reviewers who have contributed substantially to the quality of this collection.

\section{References}

Aoki, Kenichi \& Marcus W. Feldman. 1991. Recessive hereditary deafness, assortative mating, and persistence of a sign language. Theoretical Population Biology 39(3). $358-372$.

Arbib, Michael A. 2012. How the Brain got Language: The Mirror Systems Hypothesis. Oxford: Oxford University Press.

Beckner, Clay, Richard Blythe, Joan Bybee, Morten H. Christiansen, William Croft, Nick C. Ellis, John Holland, Jinyun Ke, Diane Larsen-Freeman \& Tom Schoenemann. 20og. Language is a complex adaptive system: Position paper. Language Learning 59 Suppl. 1. 1-26. doi:10.1111/j.1467-9922.2009.00533.x

Bentz, Christian, Dan Dediu, Annemarie Verkerk \& Gerhard Jäger. 2018. The evolution of language families is shaped by the environment beyond neutral drift. Nature Human Behaviour 2(11). 816-821. doi:10.1038/s41562-018-0457-6.

Bickel, Balthasar. 2015. Distributional typology: Statistical inquiries into the dynamics of linguistic diversity. In Bernd Heine \& Heiko Narrog (eds.), The Oxford Handbook of Linguistic Analysis, 901-924. Oxford: Oxford University Press. doi:10.1093/oxfordhb/ 9780199677078.013.0046.

Blasi, Damián E., Steven Moran, Scott R. Moisik, Paul Widmer, Dan Dediu \& Balthasar Bickel. 2019. Human sound systems are shaped by post-Neolithic changes in bite configuration. Science 363(6432). eaav3218. doi:10.1126/science.aav3218.

Blasi, Damián E., Søren Wichmann, Harald Hammarström, Peter F. Stadler \& Morten H. Christiansen. 2016. Sound-meaning association biases evidenced across thousands of languages. Proceedings of the National Academy of Sciences 113(39). 1081810823. doi:10.1073/pnas.1605782113.

Blevins, Juliette. 2004. Evolutionary Phonology: The Emergence of Sound Patterns. Cambridge: Cambridge University Press.

Bohnemeyer, Jürgen, Katharine T. Donelson, Randi E. Moore, Elena Benedicto, Alyson Eggleston, Carolyn K. O'Meara, Gabriela Pérez Báez, et al. 2015. The contact diffusion 
of linguistic practices. Language Dynamics and Change 5(2). 169-201. doi:10.1163/ 22105832-00502002.

Botha, Rudolf. 2012. Inferring modern language from ancient objects. In Maggie Tallerman \& Kathleen R. Gibson (eds.), The Oxford Handbook of Language Evolution, 303312. Oxford: Oxford University Press. doi:10.1093/oxfordhb/9780199541119.013.0030

Bouckaert, Remco R., Claire Bowern \& Quentin D. Atkinson. 2018. The origin and expansion of Pama-Nyungan languages across Australia. Nature Ecology \& Evolution 2(4). 741-749.

Brezina, Vaclav. 2018. Statistics in Corpus Linguistics: A Practical Guide. Cambridge University Press.

Brown, Angela M. \& Delwin T. Lindsey. 2004. Color and language: Worldwide distribution of Daltonism and distinct words for "blue." Visual Neuroscience 21(3). 409-412. doi:10.1017/So952523804213098.

Bybee, Joan. 2010. Language, usage and cognition. Cambridge: Cambridge University Press.

Caldwell, Christine A. \& Ailsa E. Millen. 2008. Experimental models for testing hypotheses about cumulative cultural evolution. Evolution and Human Behavior 29(3).165171. doi:10.1016/j.evolhumbehav.2007.12.001

Carr, Jon W., Kenny Smith, Jennifer Culbertson \& Simon Kirby. forthc. Simplicity and informativeness in semantic category systems. Cognition. Preprint: https://psyarxiv .com/jkfyx/.

Carr, Jon W., Kenny Smith, Hannah Cornish \& Simon Kirby. 2017. The cultural evolution of structured languages in an open-ended, continuous world. Cognitive Science 41(4). 892-923. doi:10.1111/cogs.12371.

Chen, M. Keith. 2013. The effect of language on economic behavior: Evidence from savings rates, health behaviors, and retirement assets. American Economic Review 103(2). 69o-731. doi:10.1257/aer.103.2.69o.

Christiansen, Morten H. \& Simon Kirby. 2003. Language evolution: The hardest problem in science. In Morten H. Christiansen \& Simon Kirby (eds.), Language Evolution (Oxford Studies in the Evolution of Language 3), 1-15. Oxford: Oxford University Press. doi:10.1093/acprof:oso/9780199244843.003.00o1

Choi, Edward, Angeliki Lazaridou \& Nando de Freitas. 2018. Compositional obverter communication learning from raw visual input. arXiv:1804.02341 [cs].

Cogswell, Michael, Jiasen Lu, Stefan Lee, Devi Parikh \& Dhruv Batra. 2019. Emergence of compositional language with deep generational transmission. arXiv:1904.09067 [cs, stat].

Corballis, Michael C. 2003. From mouth to hand: Gesture, speech, and the evolution of right-handedness. Behavioral and Brain Sciences 26(o2).199-26o. doi:10.1017/ s0140525X03000062

Corballis, Michael C. 2012. The origins of language in manual gestures. In Maggie Taller- 
man \& Kathleen R. Gibson (eds.), The Oxford Handbook of Language Evolution, 382386. Oxford: Oxford University Press. doi:10.1093/oxfordhb/9780199541119.013.0041 Coupé, Christophe, Yoon Oh, Dan Dediu \& François Pellegrino. 2019. Different languages, similar encoding efficiency: Comparable information rates across the human communicative niche. Science Advances 5(9). eaaw2594. doi:10.1126/sciadv .aaw2594.

Croft, William. 200o. Explaining Language Change: An Evolutionary Approach. Harlow: Pearson Longman.

Culbertson, J. \& David Adger. 2014. Language learners privilege structured meaning over surface frequency. Proceedings of the National Academy of Sciences 111(16). 5842-5847. doi:10.1073/pnas.1320525111.

d'Errico, Francesco \& Marian Vanhaeren. 2009. Earliest personal ornaments and their significance for the origin of language debate. In Rudolf Botha \& Chris Knight (eds.), The Cradle of Language, 16-40. Oxford: Oxford University Press.

Dediu, Dan \& Bart de Boer. 2016. Language evolution needs its own journal. Journal of Language Evolution 1(1). 1-6. doi:10.1093/jole/lzvoo1.

Dediu, Dan \& Stephen C. Levinson. 2013. On the antiquity of language: The reinterpretation of Neandertal linguistic capacities and its consequences. Frontiers in Psychology 4. doi:10.3389/fpsyg.2013.00397.

Dediu, Dan \& Stephen C. Levinson. 2018. Neanderthal language revisited: Not only us. Current Opinion in Behavioral Sciences 21. 49-55. doi:10.1016/j.cobeha.2018.01.001.

Dingemanse, Mark, Damián E. Blasi, Gary Lupyan, Morten H. Christiansen \& Padraic Monaghan. 2015. Arbitrariness, iconicity, and systematicity in language. Trends in Cognitive Sciences 19(10). 6o3-615.

Dryer, Matthew S. \& Martin Haspelmath (eds.). 2013. WALs online. Leipzig: Max Planck Institute for Evolutionary Anthropology. https://wals.info/.

Dunbar, Robin. 1996. Grooming, Gossip, and the Evolution of Language. Cambridge: Harvard University Press.

Evans, Nicholas. 2013. Language diversity as a tool for understanding cultural evolution. In Peter J. Richerson \& Morten H. Christiansen (eds.), Cultural Evolution: Society, Technology, Language, and Religion, 233-268. Cambridge: м Iт Press.

Everett, Caleb, Damián E. Blasi \& Seán G. Roberts. 2015. Climate, vocal folds, and tonal languages: Connecting the physiological and geographic dots. Proceedings of the National Academy of Sciences 112(5). 1322-1327. doi:10.1073/pnas.1417413112.

Feldmann, Horst. 2019. Do linguistic structures affect human capital? The case of pronoun drop. Kyklos 72(1). 29-54. doi:10.1111/kykl.1219o.

Fitch, W. Tecumseh. 2010. The Evolution of Language. Cambridge: Cambridge University Press.

Fitch, W. Tecumseh. 2017. Empirical approaches to the study of language evolution. Psychonomic Bulletin \& Review 24(1). 3-33. doi:10.3758/s13423-017-1236-5. 
François, Quesque \& Yves Rossetti. 2020. What do theory-of-mind tasks actually measure? Theory and practice. Perspectives on Psychological Science. doi:10.1177/ 17456916198966 o7.

Galantucci, Bruno. 2005. An experimental study of the emergence of human communication systems. Cognitive Science 29(5) · 737-767. doi:10.1207/s15516709cogoooo_34.

Galantucci, Bruno, Simon Garrod \& Gareth Roberts. 2012. Experimental semiotics. Language and Linguistics Compass 6(8). 477-493. doi:10.1002/lnc3.351.

Goldin-Meadow, Susan, Wing Chee So, Aslı Özyürek \& Carolyn Mylander. 20o8. The natural order of events: How speakers of different languages represent events nonverbally. Proceedings of the National Academy of Sciences 105(27). 9163-9168. doi:10 .1073/pnas.071006o105.

Gray, Russell D., Alexei J. Drummond \& Simon J. Greenhill. 2009. Language phylogenies reveal expansion pulses and pauses in Pacific settlement. Science 323(5913). 479483.

Hammarström, Harald. 2016. Linguistic diversity and language evolution. Journal of Language Evolution 1(1). 19-29.

Hammarström, Harald, Robert Forkel \& Martin Haspelmath. 2019. Glottolog 4.1. https:// glottolog.org/.

Hartmann, Stefan. in press. Language change and language evolution: Cousins, siblings, twins? Glottotheory.

Haspelmath, Martin. 2020. Human linguisticality and the building blocks of languages. Frontiers in Psychology 10. doi:10.3389/fpsyg.2019.03056

Heine, Bernd \& Tania Kuteva. 2007. The Genesis of Grammar: A Reconstruction. Oxford: Oxford University Press.

Heine, Bernd \& Tania Kuteva. 2012. Grammaticalization theory as a tool for reconstructing language evolution. In Maggie Tallerman \& Kathleen R. Gibson (eds.), The Oxford Handbook of Language Evolution, 511-527. Oxford: Oxford University Press.

Hua, Xia, Simon J. Greenhill, Marcel Cardillo, Hilde Schneemann \& Lindell Bromham. 2019. The ecological drivers of variation in global language diversity. Nature Communications 10(1). 2047. doi:10.1038/s41467-019-09842-2.

Humboldt, Wilhelm von. ${ }^{18} 36$. Über die Verschiedenheit des menschlichen Sprachbaues und ihren Einfluß auf die geistige Entwickelung des Menschengeschlechts. Berlin: Akademie der Wissenschaften.

Hurford, James R. 199o. Nativist and Functional Explanations in Language Acquisition. In I.M. Roca (ed.), Logical Issues in Language Acquisition, 85-136. Dordrecht: Foris.

Jackson, Joshua Conrad, Joseph Watts, Teague R. Henry, Johann-Mattis List, Robert Forkel, Peter J. Mucha, Simon J. Greenhill, Russell D. Gray \& Kristen A. Lindquist. 2019. Emotion semantics show both cultural variation and universal structure. Science 366(6472). 1517-1522. doi:10.1126/science.aaw816o. 
Kanwal, Jasmeen, Kenny Smith, Jennifer Culbertson \& Simon Kirby. 2017. Zipf's Law of Abbreviation and the Principle of Least Effort: Language users optimise a miniature lexicon for efficient communication. Cognition 165. 45-52. doi:10.1016/j.cognition .2017 .05 .001 .

Kendon, Adam. 2017. Reflections on the "gesture-first" hypothesis of language origins. Psychonomic Bulletin \& Review 24(1). 163-170.

Kim, Jaehyeon, Yongtae Kim \& Jian Zhou. 2017. Languages and earnings management. SSRN Electronic Journal. doi:10.2139/ssrn.2941120.

Kirby, Kathryn R., Russell D. Gray, Simon J. Greenhill, Fiona M. Jordan, Stephanie Gomes-Ng, Hans-Jörg Bibiko, Damián E. Blasi, et al. 2016. D-P LACE: A global database of cultural, linguistic and environmental diversity. PLOS ONE 11(7). eo158391. doi:10 $.1371 /$ journal.pone.0158391.

Kirby, Simon. 2012. Language is an adaptive system: The role of cultural evolution in the origins of structure. In Maggie Tallerman \& Kathleen R. Gibson (eds.), The Oxford Handbook of Language Evolution, 589-6o4. Oxford: Oxford University Press.

Kirby, Simon. 2017. Culture and biology in the origins of linguistic structure. Psychonomic Bulletin \& Review 24(1). 118-137. doi:10.3758/s13423-016-1166-7.

Kirby, Simon, Hannah Cornish \& Kenny Smith. 2008. Cumulative cultural evolution in the laboratory: an experimental approach to the origins of structure in human language. Proceedings of the National Academy of Sciences of the United States of America 105(31). 10681-6. doi:10.1073/pnas.0707835105.

Kirby, Simon \& James R. Hurford. 2002. The emergence of linguistic structure: An overview of the iterated learning model. In Angelo Cangelosi \& Domenico Parisi (eds.), Simulating the Evolution of Language, 121-147. London: Springer London. doi:10.1007/978-1-4471-0663-o_6.

Kirby, Simon, Monica Tamariz, Hannah Cornish \& Kenny Smith. 2015. Compression and communication in the cultural evolution of linguistic structure. Cognition 141. 87-102. doi:10.1016/j.cognition.2015.03.016.

Kirby, Simon, Tom Griffiths \& Kenny Smith. 2014. Iterated learning and the evolution of language. Current Opinion in Neurobiology 28. 108-114. doi:10.1016/j.conb.2014.07 .014 .

Lazaridou, Angeliki, Alexander Peysakhovich \& Marco Baroni. 2017. Multi-agent cooperation and the emergence of (natural) language. arXiv:1612.07182 [cs].

Little, Hannah, Kerem Eryılmaz \& Bart de Boer. 2017. Signal dimensionality and the emergence of combinatorial structure. Cognition 168. 1-15. doi:10.1016/j.cognition .2017.06.011.

Lupyan, Gary \& Rick Dale. 2010. Language structure is partly determined by social structure. (Ed. Dennis O'Rourke). PLOS ONE 5(1). e8559. doi:10.1371/journal.pone .0008559 .

Lupyan, Gary \& Rick Dale. 2016. Why are there different languages? The role of adapta- 
tion in linguistic diversity. Trends in Cognitive Sciences 20(9). 649-66o. doi:10.1016/j .tics.2016.07.005.

MacNeilage, Peter F. 2008. The Origin of Speech. (Studies in the Evolution of Language 10). Oxford: Oxford University Press.

Macuch Silva, Vinicius, Judith Holler, Asli Ozyurek \& Seán G. Roberts. 2020. Multimodality and the origin of a novel communication system in face-to-face interaction. Royal Society Open Science 7(1).

Majid, Asifa, Melissa Bowerman, Sotaro Kita, Daniel B.M. Haun \& Stephen C. Levinson. 2004. Can language restructure cognition? The case for space. Trends in Cognitive Sciences 8(3). 108-114. doi:10.1016/j.tics.2004.01.003.

Majid, Asifa, Seán G. Roberts, Ludy Clissen, Karen Emmorey, Brenda Nicodemus, Lucinda O'Grady, Bencie Woll, et al. 2018. Differential coding of perception in the world's languages. Proceedings of the National Academy of Sciences 115(45). 1136911376. https://doi.org10.1073/pnas.1720419115.

Martin, Alexander, Theeraporn Ratitamkul, Klaus Abels, David Adger \& Jennifer Culbertson. 2019. Cross-linguistic evidence for cognitive universals in the noun phrase. Linguistics Vanguard 5(1). doi:10.1515/lingvan-2018--oo72.

McNeill, David. 2012. How Language Began: Gesture and Speech in Human Evolution. Cambridge: Cambridge University Press.

Meir, Irit, Mark Aronoff, Carl Börstell, So-One Hwang, Deniz Ilkbasaran, Itamar Kastner, Ryan Lepic, Adi Lifshitz Ben-Basat, Carol Padden \& Wendy Sandler. 2017. The effect of being human and the basis of grammatical word order: Insights from novel communication systems and young sign languages. Cognition 158. 189-207. doi:10 .1016/j.cognition.2016.10.011.

Mendívil-Giró, José-Luis. 2019. Did language evolve through language change? On language change, language evolution and grammaticalization theory. Glossa: A Journal of General Linguistics 4(1).

Michel, Jean-Baptiste, Yuan Kui Shen, Aviva Presser Aiden, Adrian Veres, Matthew K. Gray, The Google Books Team, Joseph P. Pickett, Dale Hoiberg, Dan Clancy, Peter Norvig, Jon Orwant, Steven Pinker, Martin A. Nowak \& Erez Lieberman Aiden. 2011. Quantitative Analysis of Culture Using Millions of Digitized Books. Science 331(6o14). 176-182.

Moisik, Scott Reid \& Dan Dediu. 2017. Anatomical biasing and clicks: Evidence from biomechanical modeling. Journal of Language Evolution 2(1).37-51. doi:10.1093/jole/ lzxoo4.

Moran, Steven \& Daniel McCloy (eds.). 2019. РноІвLE 2.o. Jena: Max Planck Institute for the Science of Human History. https://phoible.org/.

Mordatch, Igor \& Pieter Abbeel. 2018. Emergence of grounded compositional language in multi-agent populations. In Thirty-Second AAAI Conference on Artificial Intelligence Proceedings. 1495-1503. 
Motamedi, Yasamin, Hannah Little, Alan Nielsen \& Justin Sulik. 2019a. The iconicity toolbox: Empirical approaches to measuring iconicity. Language and Cognition 11(02). 188-207. doi:10.1017/langcog.2019.14.

Motamedi, Yasamin, Marieke Schouwstra, Kenny Smith, Jennifer Culbertson \& Simon Kirby. 2019b. Evolving artificial sign languages in the lab: From improvised gesture to systematic sign. Cognition 192. 103964. doi:10.1016/j.cognition.2019.05.001.

Müller, Cornelia, Alan J. Cienki, Ellen Fricke, Silva H. Ladewig, David McNeill \& Sedinha Tessendorf (eds.). 2013. Body -Language-Communication: An International Handbook on Multimodality in Human Interaction. Berlin, Boston: De Gruyter Mouton.

Müller, Thomas F. \& James Winters. 2018. Compression in cultural evolution: Homogeneity and structure in the emergence and evolution of a large-scale online collaborative art project. (Ed.) Alex Mesoudi. PLOS ONE 13(9). eo2O2O19.

Nölle, Jonas, Marlene Staib, Riccardo Fusaroli \& Kristian Tylén. 2018. The emergence of systematicity: How environmental and communicative factors shape a novel communication system. Cognition 181. 93-104.

Nölle, Jonas, Riccardo Fusaroli, Gregory J. Mills \& Kristian Tylén. 2020a. Language as shaped by the environment: Linguistic construal in a collaborative spatial task. Palgrave Communications 6(1). 27. doi:10.1057/s41599-020-0404-9.

Nölle, Jonas, Simon Kirby, Jennifer Culbertson \& Kenny Smith. 2020b. Does environment shape spatial language? A virtual reality experiment. In Andrea Ravignani, Chiara Barbieri, Mauricio Martins, Molly Flaherty, Yannick Jadoul, Ella Lattenkamp, Hannah Little, Katie Mudd \& Tessa Verhoef (eds.), The Evolution of Language: Proceedings of the 13 th International Conference (EvoLang13). doi:10.17617/2.3190925.

Open Science Collaboration. 2015. Estimating the reproducibility of psychological science. Science 349(6251). aac4716-aac4716. doi:10.1126/science.aac4716.

Özçalışkan, Şeyda, Ché Lucero \& Susan Goldin-Meadow. 2016. Does language shape silent gesture? Cognition 148. 10-18. doi:10.1016/j.cognition.2015.12.001.

Palmer, Bill, Jonathon Lum, Jonathan Schlossberg \& Alice Gaby. 2017. How does the environment shape spatial language? Evidence for sociotopography. Linguistic Typology 21(3). doi:10.1515/lingty-2017-oo11.

Pavlek, Barbara, James Winters \& Olivier Morin. 2019. Ancient coin designs encoded increasing amounts of economic information over centuries. Journal of Anthropological Archaeology 56. 101103 .

Pearl, Judea. 20o9. Causality: Models, Reasoning and Inference. 2nd edition. New York: Cambridge University Press.

Peeters, David. 2019. Virtual reality: A game-changing method for the language sciences. Psychonomic Bulletin \& Review 26(3). 894-9oo. doi:10.3758/s13423-019-01571-3.

Perlman, Marcus. 2017. Debunking two myths against vocal origins of language: Language is iconic and multimodal to the core. Interaction Studies 18(3). 376-401.

Perlman, Marcus, Hannah Little, Bill Thompson \& Robin L. Thompson. 2018. Iconicity 
in signed and spoken vocabulary: A comparison between American Sign Language, British Sign Language, English, and Spanish. Frontiers in Psychology 9. 1433. doi:10 .3389/fpsyg.2018.01433.

Perniss, Pamela \& Gabriella Vigliocco. 2014. The bridge of iconicity: From a world of experience to the experience of language. Philosophical Transactions of the Royal Society B: Biological Sciences 369(1651). doi:10.1098/rstb.2013.0300 (5 May, 2017).

Perniss, Pamela, Robin L. Thompson \& Gabriella Vigliocco. 2010. Iconicity as a general property of language: Evidence from spoken and signed languages. Frontiers in Psychology 1. doi:10.3389/fpsyg.2010.00227 (2 May, 2019).

Petré, Peter \& Freek Van de Velde. 2018. The real-time dynamics of the individual and the community in grammaticalization. Language 94(4). 867-901. doi:10.1353/ lan.2018.0056.

Pleyer, Michael, Stefan Hartmann, James Winters \& Jordan Zlatev. 2017. Interaction and iconicity in the evolution of language: Introduction to the special issue. Interaction Studies 18(3). 303-313.

Raviv, Limor, Antje Meyer \& Shiri Lev-Ari. 2019. Larger communities create more systematic languages. Proceedings of the Royal Society B: Biological Sciences 286(1907). 20191262. doi:10.1098/rspb.2019.1262.

Regier, Terry, Alexandra Carstensen \& Charles Kemp. 2016. Languages support efficient communication about the environment: words for snow revisited. (Ed. Thomas Wennekers). PLOS ONE 11(4). eo151138. doi:10.1371/journal.pone.0151138.

Ren, Yi, Shangmin Guo, Serhii Havrylov, Shay Cohen \& Simon Kirby. 2019. Enhance the Compositionality of Emergent Language by Iterated Learning. 3rd NeurIPS Workshop on Emergent Communication (EmeCom @ NeurIPS 2019). URL https://papers .nips.cc/book/advances-in-neural-information-processing-systems-32-2019.

Ritt, Nikolaus. 2004. Selfish Sounds and Linguistic Evolution: A Darwinian Approach to Language Change. Cambridge: Cambridge University Press.

Roberts, Gareth. 2017. The linguist's Drosophila: Experiments in language change. Linguistics Vanguard 3(1). doi:10.1515/lingvan-2016--oo86.

Roberts, Gareth \& Maryia Fedzechkina. 2018. Social biases modulate the loss of redundant forms in the cultural evolution of language. Cognition 171. 194-201. doi:10.1016/ j.cognition.2017.11.005.

Roberts, Gareth, Jirka Lewandowski \& Bruno Galantucci. 2015. How communication changes when we cannot mime the world: Experimental evidence for the effect of iconicity on combinatoriality. Cognition 141. 52-66. doi:10.1016/j.cognition.2015.04 .oo1.

Roberts, Seán G. 2018a. CHIELD: Causal hypotheses in evolutionary linguistics database. In Christine Cuskley, Molly Flaherty, Luke McCrohon, Hannah Little, Andrea Ravignani \& Tessa Verhoef (eds.), The Evolution of Language. Proceedings of the 12th International Conference, 412-418. Torun: Nicolaus Copernicus University. 
Roberts, Seán G. 2018b. Robust, Causal, and Incremental Approaches to Investigating Linguistic Adaptation. Frontiers in Psychology 9. doi:10.3389/fpsyg.2018.oo166.

Roberts, Seán \& James Winters. 2012. Social Structure and Language Structure: The New Nomothetic Approach. Psychology of Language and Communication 16(2). 89-112.

Roberts, Seán \& James Winters. 2013. Linguistic Diversity and Traffic Accidents: Lessons from Statistical Studies of Cultural Traits. PLOS ONE 8(8). doi:10.1371/journal.pone .0070902.

Roberts, Seán G., James Winters \& Keith Chen. 2015. Future Tense and Economic Decisions: Controlling for Cultural Evolution. (Ed.) Ramesh Balasubramaniam. PLOS ONE 1O(7). eo132145. doi:10.1371/journal.pone.0132145.

Roberts, Seán G., Angarika Deb, Catherine Sheard, Simon J. Greenhill, Kaius Sinnemäki, José Segovia-Martín, Jonas Nölle, Aleksandrs Berdicevskis, Archie Humphreys-Balkwill, Hannah Little, Kit Opie, Guillaume Jacques, Lindell Bromham, Peeter Tinits, Robert M. Ross, Sean Lee, Emily Gasser, Jasmine Calladine, Matthew Spike, et al. forthc. CHIELD: The causal hypotheses in Evolutionary Linguistics Database. Journal of Language Evolution 5(1).

Roberts, Seán G., Anton Killin, Angarika Deb, Catherine Sheard, Simon J. Greenhill, Kaius Sinnemäki, José Segovia-Martín, et al. 2020. CHIELd: The causal hypotheses in evolutionary linguistics database. Journal of Language Evolution 5(1). https://doi.org10.1093/jole/lzaaoo1. https://academic.oup.com/jole/article/doi/10.10 93/jole/lzaaoo1/5821004.

Schouwstra, Marieke \& Henriëtte de Swart. 2014. The semantic origins of word order. Cognition 131(3). 431-436. doi:10.1016/j.cognition.2014.03.004.

Shapero, Joshua A. 2017. Does Environmental experience shape spatial cognition? Frames of reference among Ancash Quechua speakers (Peru). Cognitive Science 41(5). 1274-1298. doi:10.1111/cogs.12458.

Sneller, Betsy \& Gareth Roberts. 2018. Why some behaviors spread while others don't: A laboratory simulation of dialect contact. Cognition 170. 298-311. doi:10.1016/j.cogni tion.2017.10.014.

Spranger, Michael 2016. The Evolution of Grounded Spatial Language. Berlin: Language Science Press.

Steels, Luc. 1995. A self-organizing spatial vocabulary. Artificial Life 2(3). 319-332.

Steels, Luc. 200o. Language as a complex adaptive system. In Marc Schoenauer, Kalyanmoy Deb, Günter Rudolph, Xin Yao, Evelyne Lutton, Juan Julian Merelo \& HansPaul Schwefel (eds.), Parallel Problem Solving from Nature, 17-26. Berlin, Heidelberg: Springer.

Stevens, Jon S. \& Gareth Roberts. 2019. Noise, economy, and the emergence of information structure in a laboratory language. Cognitive Science 43(2). e12717. doi:10.1111/ cogs.12717.

Sulik, Justin \& Gary Lupyan. 2020. Appeals to theory of mind no longer explain much in 
language evolution. In Andrea Ravignani, Chiara Barbieri, Mauricio Martins, Molly Flaherty, Yannick Jadoul, Ella Lattenkamp, Hannah Little, Katie Mudd \& Tessa Verhoef (eds.), The Evolution of Language: Proceedings of the 13th International Conference.

Syrjänen, Kaj, Terhi Honkola, Jyri Lehtinen, Antti Leino \& Outi Vesakoski. 2016. Applying population genetic approaches within languages. Language Dynamics and Change 6(2). 235-283.

Tallerman, Maggie \& Kathleen R. Gibson (eds.). 2012. The Oxford Handbook of Language Evolution. Oxford: Oxford University Press.

Tamariz, Monica. 2017. Experimental studies on the cultural evolution of language. Annual Review of Linguistics 3(1). 389-407. doi:10.1146/annurev-linguistics-011516 -033807.

Tinbergen, N. 1963. On aims and methods of ethology. Zeitschrift für Tierpsychologie 2o(4). 410-433. doi:10.1111/j.1439--0310.1963.tbo1161.x.

Tinits, Peeter, Jonas Nölle \& Stefan Hartmann. 2017. Usage context influences the evolution of overspecification in iterated learning. Journal of Language Evolution 2(2). 148-159. doi:10.1093/jole/lzxo11.

Tomasello, Michael. 2008. Origins of Human Communication. Cambridge: MIT Press.

Torreira, Francisco, Sara Bögels \& Stephen C. Levinson. 2015. Breathing for answering: The time course of response planning in conversation. Frontiers in Psychology 6. doi:10.3389/fpsyg.2015.00284 (6 January, 2020).

Tylén, Kristian, Riccardo Fusaroli, Sergio Rojo, Katrin Heimann, Nicolas Fay, Niels N. Johannsen, Felix Riede \& Marlize Lombard. 2020. The evolution of early symbolic behavior in Homo sapiens. Proceedings of the National Academy of Sciences. doi:10.1073/pnas.191088o117.

Wacewicz, Sławomir \& Przemyslaw Żywiczyński. 2017. The multimodal origins of linguistic communication. Language \& Communication 54. 1-8.

Wassmann, Jurg \& Pierre R. Dasen. 1998. Balinese spatial orientation: Some empirical evidence of moderate linguistic relativity. Journal of the Royal Anthropological Institute 4(4). 689+.

Weinreich, Uriel, Labov, William, \& Herzog, Marvin L. 1968. Empirical foundations for a theory of language change. In Winfred P. Lehmann \& Yakov Malkiel (eds.), Directions for historical linguistics: a symposium, 95-195. Austin: University of Texas Press.

Wichmann, Søren, Eric W. Holman, and Cecil H. Brown (eds.). 2018. The AsJ P Database (version 18).

Winter, Bodo, Marcus Perlman \& Asifa Majid. 2018. Vision dominates in perceptual language: English sensory vocabulary is optimized for usage. Cognition 179. 213-220. doi:10.1016/j.cognition.2018.05.008.

Winters, James, Simon Kirby \& Kenny Smith. 2015. Languages adapt to their contextual niche. Language and Cognition 7(3). 415-449. doi:10.1017/langcog.2014.35. 
Winters, James, Simon Kirby \& Kenny Smith. 2018. Contextual predictability shapes signal autonomy. Cognition 176. 15-30. doi:10.1016/j.cognition.2018.o3.0o2.

Woensdregt, Marieke, Chris Cummins \& Kenny Smith. forthc. A computational model of the cultural co-evolution of language and mindreading. Synthese. Preprint: https://psyarxiv.com/3bmsx/

Zlatev, Jordan, Sławomir Wacewicz, Przemysław Żywiczyński \& Joost van de Weijer. 2017. Multimodal-first or pantomime-first? Communicating events through pantomime with and without vocalization. Interaction Studies 18(3). 465-488.

Żywiczyński, Przemysław, Sławomir Wacewicz \& Marta Sibierska. 2018. Defining pantomime for language evolution research. Topoi 37(2). 307-318. 